\title{
UV Light-emitting Diode with Buried Polarization-induced n-AlGaN/InGaN/p-AlGaN Tunneling Junction
}

\author{
Yi Lu, Chuanju Wang, Victor Paiva De Oliveira, Zhiyuan Liu, and Xiaohang Li
}

\begin{abstract}
The polarization-induced electric field in the IIInitride UV light-emitting diode (LED) allows for significant flexibility in device design to address the electron overflow and hole injection issues. The conventional AIGaN-based UV LED with the PIN structure suffers from insufficient carriers especially hole concentration due to the large valence band barrier for hole injection and p-type doping challenge. Our systematic study reveals that the inverse design of the n-type and p-type layer shall build an opposite polarization-induced field to suppress electron overflow as well as simultaneously enhance hole injection. To design this p-side down UV LED and improve the hole injection, we adopt the n-AlGaN/i-InGaN/p-AlGaN buried tunneling junction (BTJ) instead of the bottom p-layer. The tunneling probability and output power of the LED are further investigated by optimizing the composition and thickness of the InGaN layer. Simulation results show that the optimized $3 \mathrm{~nm} \operatorname{In}_{0.3} \mathrm{Ga}_{0.7} \mathrm{~N}$ tunneling layer could lead to several orders of magnitude enhancement for LED output power. This study is significant for the pursuit of highly efficient UV LEDs.
\end{abstract}

Index Terms-Aluminum gallium nitride, Light emitting diodes, Buried tunneling junction, Ultraviolet sources, p-down LED

\section{INTRODUCTION}

$\mathrm{T}$ he AlGaN-based UV LED has been proven to be a promising light source to replace the mercury lamp for disinfection, communication, curing, and bio-medical detection [1]. Compared with the traditional UV light sources, the AlGaN-based UV LED has the strengths of compactness, portability, pro-environment, and long-lasting [2]. However, the commercial applications of UV LED are still limited due to the remaining technical challenges for further improving the external quantum efficiency (EQE).

The UV LED generally starts from the n-type AlGaN template on a template or substrate, followed by the active region, and then p-layer. This PIN structure grown along the (0001) direction could be described as the "III-polar, p-up" structure. However, some reports have proven that if growing this PIN structure with the nitrogen-polar (N-polar, p-up) orientation, the EQE could be further improved, stemming from

This work was supported by the KAUST Baseline BAS/1/1664-01-01, KAUST CRG URF/1/3437-01-01, GCC REP/1/3189-01-01, and National Natural Science Foundation of China (Grant No.61774065).

Y. Lu, C. Wang, V. P. Oliveira, Z. Liu, and X. Li are with the Advanced Semiconductor Laboratory, King Abdullah University of Science and the desirable barriers for better carrier injection and confinement [3, 4]. However, the N-polar materials are still immature due to the epitaxial difficulties [5, 6]. An alternative method is to turn over the polarity and the epitaxy order simultaneously, which is to have the III-polar orientation but grow the p-layer first, then active region and n-layer. This "IIIpolar, p-down" design could provide the same polarizationinduced electric field as the "N-polar, $\mathrm{p}$-up" structure for carrier transport. The formed inverse polarization field could lower the carrier injection barrier, as well as form the band offset barrier for better carrier confinement, thus enhancing the carrier concentration and EQE. The band diagrams and potential barriers for comparing these LED configurations are shown in Fig. S1 and Table SI in the Supplementary Material (SM).

The efficient LED with the aforementioned "III-polar, pdown" structure has been demonstrated in blue and green regions by different growth techniques including metalorganic chemical vapor deposition (MOCVD), molecular beam epitaxy (MBE), and hydride vapor phase epitaxy (HVPE). To improve the hole injection, the band-to-band tunneling junction (TJ) with the $\mathrm{n}^{+} \mathrm{GaN} / \mathrm{InGaN} / \mathrm{p}-\mathrm{GaN}$ structure was specifically designed for the p-layer [7, 8]. The single heterostructure with $\mathrm{p}-\mathrm{GaN} / \mathrm{n}-\mathrm{InGaN}$ also showed visible light emission [9]. To eliminate the $\mathrm{Mg}$ memory effect in the active region after the pdoping layer, the in-situ annealing was performed $[10,11]$. Recently, the experimental study in blue and green LEDs showed that the "III-polar, p-down" with an $\mathrm{n}^{+}-\mathrm{GaN} / \mathrm{InGaN} / \mathrm{p}$ GaN BTJ could assist the carrier injection and suppress the parasitic carrier recombination [12].

The previous studies of p-down LED all focused on the blue or green region. However, the p-down design shall be employed in the UV region to enhance the carrier injection and confinement due to the more serious p-doping difficulty in AlGaN. In particular, the high-mobility electrons could easily overshoot into the p-layer, resulting in the low recombination efficiency in the active region [13]. If not designed well, the overflown electrons could even recombine with the holes in the p-layer, leading to undesired photon emission. For the "IIIpolar, p-down" structure, due to the inverse polarization field

Technology (KAUST), Thuwal 23955-6900, Saudi Arabia (email: yi.lu@kaust.edu.sa; victor.paivadeoliveira@kaust.edu.sa; xiaohang.li@kaust.edu.sa) chuanju.wang@kaust.edu.sa; zhiyuan.liu@kaust.edu.sa; 
that can form a natural electron-blocking-layer (EBL), we may remove the conventional Al\%-rich AlGaN EBL which has the p-doping challenging. That means the EBL-free UV LED could be realized through not only the composition grading in the quantum barrier [14], but also the p-down design.

In this study, we numerically investigate the $\mathrm{n}-\mathrm{AlGaN} / \mathrm{i}$ InGaN/p-AlGaN BTJ instead of the bottom p-layer to improve the carrier concentration especially the hole injection from the bottom side to the active region in the p-down UV LED. The impacts of InGaN composition and thickness which both determine the tunneling probability are studied. The device output performance shows consistency with the calculated tunneling probability. The output power of BTJ UV LED is enhanced by several orders of magnitude through the optimized $3 \mathrm{~nm} \mathrm{In} \operatorname{In}_{0.3} \mathrm{Ga}_{0.7} \mathrm{~N}$ tunneling layer.

\section{TUNNELING THEORY}

The designed "III-polar, p-down" UV LED contains a BTJ, i.e. an $\mathrm{n}-\mathrm{Al}_{0.3} \mathrm{Ga}_{0.7} \mathrm{~N} / \mathrm{i}-\mathrm{In}_{\mathrm{x}} \mathrm{Ga}_{1-\mathrm{x}} \mathrm{N} / \mathrm{p}-\mathrm{Al}_{0.3} \mathrm{Ga}_{0.7} \mathrm{~N}$ polarization $\mathrm{TJ}$, then three pairs $\mathrm{Al}_{0.3} \mathrm{Ga}_{0.7} \mathrm{~N} / \mathrm{Al}_{0.2} \mathrm{Ga}_{0.8} \mathrm{~N} / \mathrm{Al}_{0.3} \mathrm{Ga}_{0.7} \mathrm{~N}$ MQWs emitting at $\sim 320 \mathrm{~nm}$, following by an $\mathrm{n}-\mathrm{Al}_{0.3} \mathrm{Ga}_{0.7} \mathrm{~N}$ contact layer. For the $\mathrm{i}-\operatorname{In}_{\mathrm{x}} \mathrm{Ga}_{1-\mathrm{x}} \mathrm{N}$ tunneling layer, the composition $\mathrm{x}$ varies from 0 to 1 , and the thickness increases from 2 to $10 \mathrm{~nm}$ with the $1 \mathrm{~nm}$ step. Detailed information of the designed LED could be found in Fig. S2 in the SM.

The tunneling probability through the TJ could be described by (1)-(3) [15]. $T_{n}$ and $T_{p}$ are the probabilities of intraband tunneling in $\mathrm{n}-\mathrm{AlGaN}$ and $\mathrm{p}-\mathrm{AlGaN}$, respectively (corresponding to the tunneling probabilities through the Barrier 1 and 3, respectively in Fig. S3 of the SM). From the definition, $T_{n}$ and $T_{p}$ are related to the conduction band $\left(\Delta \mathrm{E}_{\mathrm{c}}\right)$ and valence band discontinuity $\left(\Delta \mathrm{E}_{\mathrm{v}}\right)$, which determine the depletion width in the $\mathrm{n}$ and p-type layers. The probability of interband tunneling through the InGaN barrier (corresponding to the tunneling probability through the Barrier 2 in Fig. S3 of the SM) is estimated based on the Kane model [16]. The "critical" thickness $\left(t_{\text {cr }}\right)$ of InGaN is defined in [17], which is related to the polarization charge difference and InGaN bandgap $\left(E_{g}\right)$ in (4). When $t<t_{c r}$, the tunneling probability is low due to the misalignment between conduction and valence band, which is corresponding to the low overlap between conduction and valence bands. When $t>t_{c r}$, the tunneling probability also reduces due to the increased thickness of the Barrier 2. The overall tunneling probability $\left(\mathrm{T}_{\text {net }}\right)$ of the polarization-induced TJ shown in (5) could be calculated by multiplying the three individual probabilities.

$$
\begin{aligned}
& T_{n}=\exp \left\{-2 \int_{0}^{x_{n}} \sqrt{\frac{m_{e}^{*} q^{2} N_{D} t^{2}}{\hbar^{2} \varepsilon}} d t\right\}, \quad x_{n}=\sqrt{\frac{2 \varepsilon \Delta E_{C}}{q^{2} N_{D}}} \\
& T_{p}=\exp \left\{-2 \int_{0}^{x_{p}} \sqrt{\frac{m_{h}^{*} q^{2} N_{A} t^{2}}{\hbar^{2} \varepsilon}} d t\right\}, \quad x_{p}=\sqrt{\frac{2 \varepsilon \Delta E_{V}}{q^{2} N_{A}}} \\
& T_{\text {InGaN }}=
\end{aligned}
$$

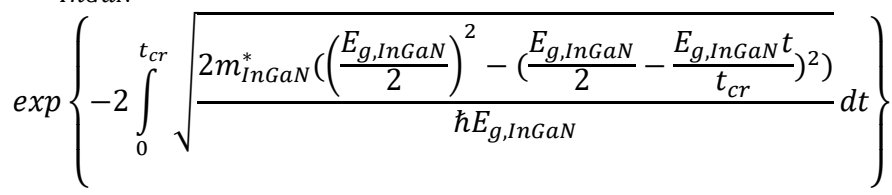

$$
\begin{gathered}
t_{c r}=\frac{E_{g} \varepsilon(x)}{q \sigma(x)} \\
T_{\text {net }}=T_{n} * T_{p} * T_{\text {InGaN }}
\end{gathered}
$$

\section{Result AND DISCUSSION}

\section{A. Effect of InGaN thickness}

Due to the difficulty of growing high-quality In-rich InGaN [18], we fix the indium composition (In\%) by 0.2 , which is realizable from the experiment report [19]. According to Fig. S4 (a) in the SM, we could extract the $t_{\text {cr }}$ under $\operatorname{In} \%=0.2$ which is $4.5 \mathrm{~nm}$. First, the n-AlGaN/i-InGaN/p-AlGaN TJs with different InGaN thicknesses from 2 to $10 \mathrm{~nm}$ are simulated under reverse bias in Fig 1. (a). JV curves show that the InGaN at $\mathrm{t}_{\mathrm{cr}}(4.5 \mathrm{~nm})$ has the minimum resistance. Then the TJs are incorporated into the UV LED (see the energy band diagrams of the BTJ UV LEDs in Fig. S4 (c) in the SM). From Fig. 1 (b), it could be observed that the emission wavelength is $\sim 320 \mathrm{~nm}$, and the highest value for spontaneous emission rate $\left(\mathrm{R}_{\mathrm{sp}}\right)$ is located at around $4.5 \mathrm{~nm}$. With higher $\mathrm{R}_{\mathrm{sp}}$, the peak wavelength shows a blue shift, which could be attributed to the reduced quantum-confined stark effect (QCSE) that comes from the more carrier injection into the active region.
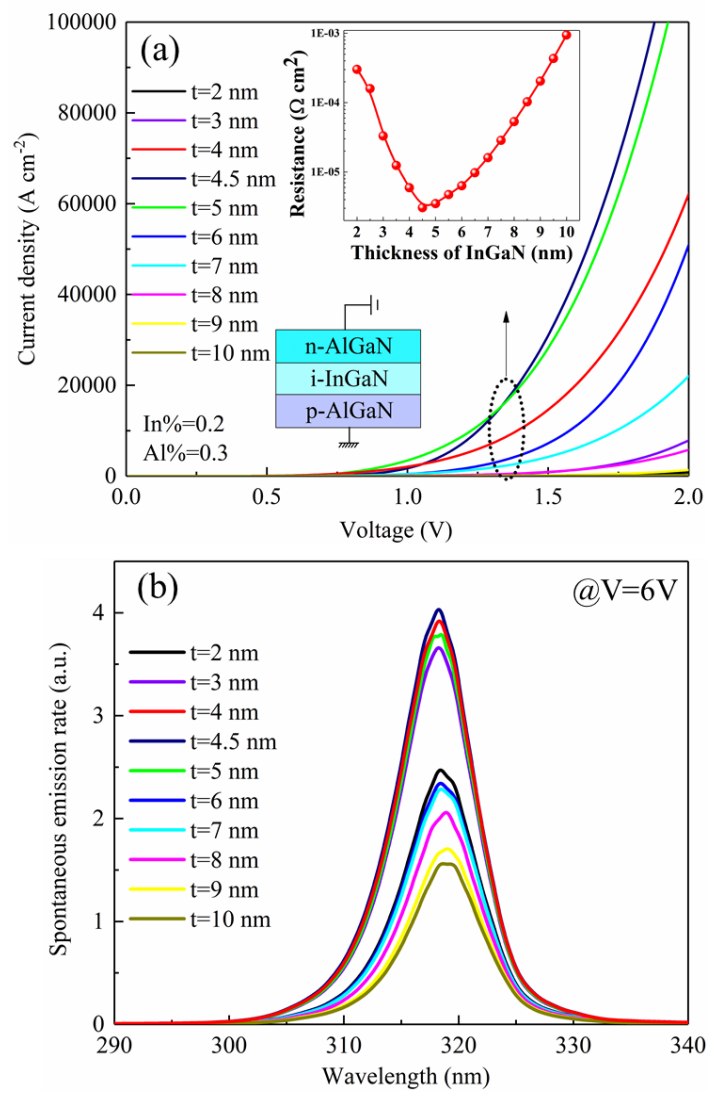

Fig. 1. (a) The current density-voltage (JV) curves of $n-A l G a N / i-I n G a N / p-$ AlGaN TJs with InGaN thickness from 2 to $10 \mathrm{~nm}$. $\mathrm{t}=2 \mathrm{~nm}, 9 \mathrm{~nm}, 10 \mathrm{~nm}$ curves are near the x-axis. Inset shows the extracted resistance from the JV curves. (b) the spontaneous emission rate spectrum $\mathrm{R}_{\mathrm{sp}}$ with different $\mathrm{InGaN}_{\mathrm{N}}$ thicknesses. The result of $4.5 \mathrm{~nm} \mathrm{InGaN}$ is also put as the comparison.

To further analyze the trends of $R_{s p}$ and output power when changing InGaN thicknesses, we extract the peak value of $R_{\text {sp }}$ 
and output power of the UV LEDs under 6V. From Fig. 2 (a), when fitting a certain In\%, with the increase of InGaN thickness, the $\mathrm{R}_{\mathrm{sp}}$ and output power would both increase first and then drop. These trends could be attributed to that the InGaN thickness which is less than and more than $t_{\text {cr }}$ would result in the $\mathrm{E}_{\mathrm{c}}-\mathrm{E}_{\mathrm{v}}$ misalignment and larger Barrier 2, respectively, as shown in the inset of Fig. 2 (a) and Fig. S3 in the SM. It could be found that there is a turning point at $\sim 4.5$ $\mathrm{nm}$ for the fitting curves, which means that the tunneling probability also reaches a maximum at $\mathrm{t}=4.5 \mathrm{~nm}$. Fig. 2 (b) provides detailed information of electron concentration $\left[\mathrm{N}_{\mathrm{QW}}\right]$, hole concentration $\left[\mathrm{P}_{\mathrm{QW}}\right]$, and radiative recombination rate $\left(\mathrm{R}_{\mathrm{rad}}\right)$ distribution in $\mathrm{QW}$ for different $\mathrm{InGaN}$ thicknesses. The highest values of $\left[\mathrm{N}_{\mathrm{QW}}\right],\left[\mathrm{P}_{\mathrm{QW}}\right]$, and $\mathrm{R}_{\mathrm{rad}}$ also happen at $\mathrm{t}_{\mathrm{cr}}(4.5$ $\mathrm{nm})$ due to the enhanced tunneling probability. The shift of the peak position of $\left[\mathrm{N}_{\mathrm{Qw}}\right],\left[\mathrm{P}_{\mathrm{QW}}\right]$, and $\mathrm{R}_{\mathrm{rad}}$ is due to the change of InGaN thickness. The spatial separation between the peaks of $\left[\mathrm{N}_{\mathrm{Qw}}\right]$ and $\left[\mathrm{P}_{\mathrm{Qw}}\right]$ in Fig. 2 (b) also indicates the presence of QCSE in the nitride-based LED.
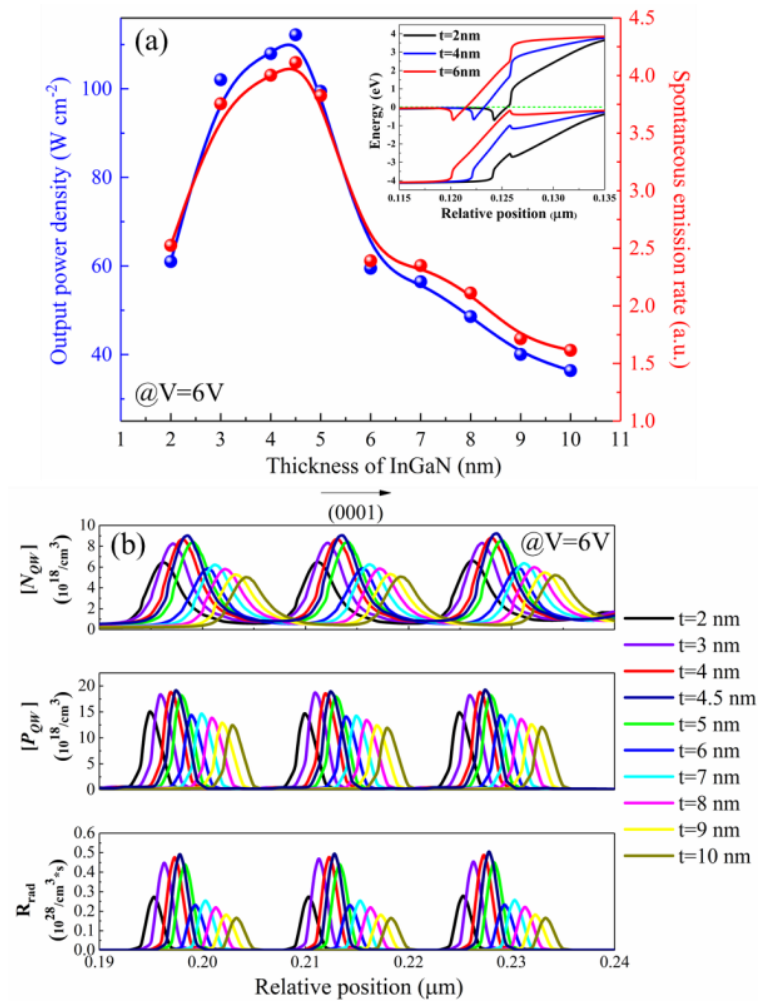

Fig. 2. (a) Output power density and peak $R_{s p}$ for different $I n G a N$ thicknesses at $6 \mathrm{~V}$. The inset is the band diagrams of $\mathrm{n}-\mathrm{AlGaN} / \mathrm{i}-\mathrm{InGaN} / \mathrm{p}-\mathrm{AlGaN}$ TJs with $2 \mathrm{~nm}, 4 \mathrm{~nm}$, and $6 \mathrm{~nm} \mathrm{InGaN}$. (b) the electron concentration $\left[\mathrm{N}_{\mathrm{QW}}\right]$, hole concentration $\left[\mathrm{P}_{\mathrm{QW}}\right]$, and radiative recombination rate $\left[\mathrm{R}_{\mathrm{rad}}\right]$ distribution in $\mathrm{QWs}$ for different InGaN thicknesses.

\section{B. Effect of InGaN composition}

To investigate the impact of InGaN composition on the BTJ LED, the In\% are varied with $0.1,0.2,0.3$, and 0.6 , and the corresponding $t_{\text {cr }}$ are $7.5 \mathrm{~nm}, 4.5 \mathrm{~nm}, 3 \mathrm{~nm}$, and $1 \mathrm{~nm}$, respectively, as shown in Fig. 3 (a). The $t_{\text {cr }}$ decreases with the increasing In\%, resulting from the enlarged polarization difference on InGaN/AlGaN interface shown in Table SII in the $\mathrm{SM}$. The increase of In\% also makes the $\Delta \mathrm{E}_{\mathrm{c}}$ and $\Delta \mathrm{E}_{\mathrm{v}}$ larger, leading to a decrease of $T_{n}$ and $T_{p}$. As a result, the $T_{n e t}$ shows an increase at the low In\% region till In $\%=0.3$, then decreases at In $\%=0.6$ from Fig. 3 (b). It could also be observed in Fig. 3 (c) that the TJ with In $\%=0.3$ has the lowest resistance, resulting from the highest $T_{\text {net }}$. In comparison, In $\%=0.1$ has the lowest tunneling probability, leading to the highest resistance among all the samples.
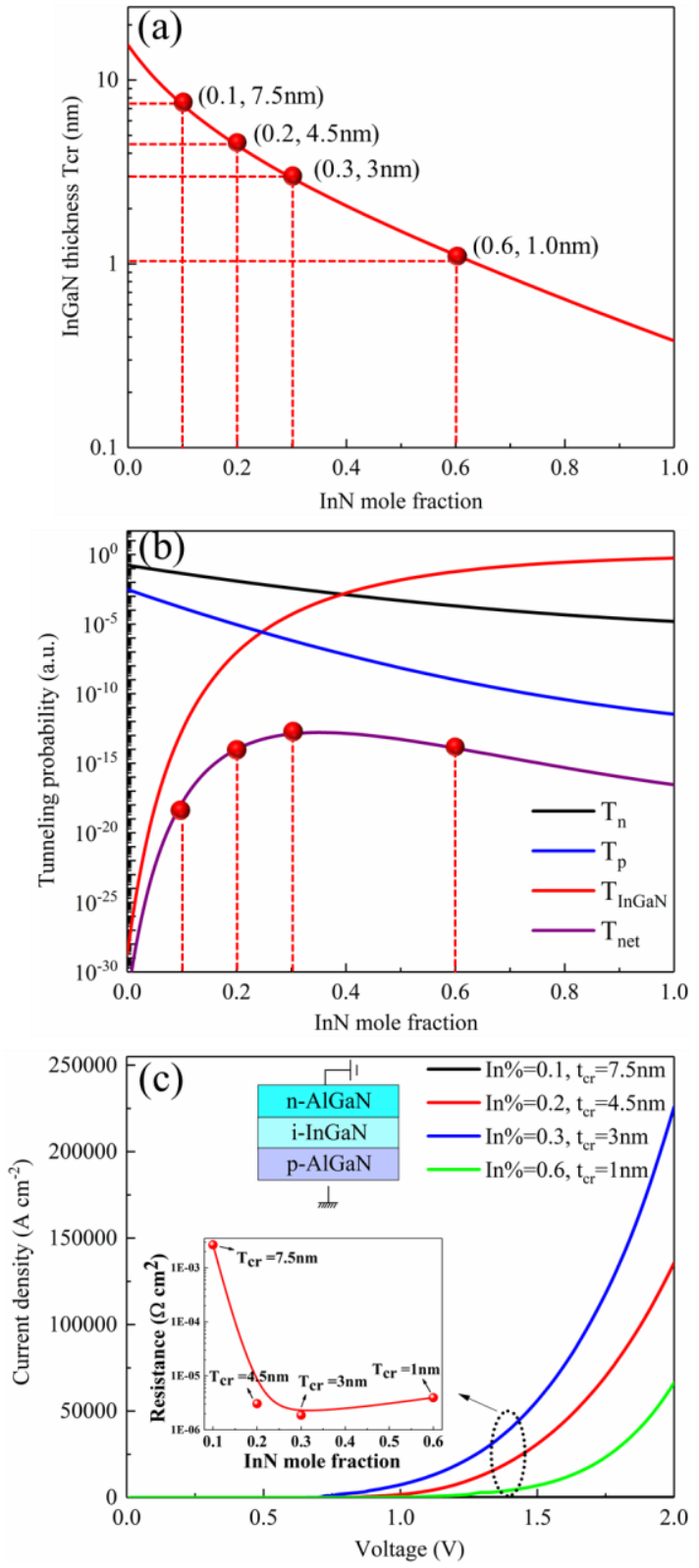

Fig. 3. (a) $t_{\mathrm{cr}}$ as a function of $\operatorname{In} \%$. In $\%=0.1,0.2,0.3$, and 0.6 are marked with the red dots. (b) tunneling probability $\mathrm{T}_{\mathrm{n}}, \mathrm{T}_{\mathrm{p}}, \mathrm{T}_{\mathrm{InGaN}}$, and $\mathrm{T}_{\text {net }}$ under different In $\%$. In $\%=0.1,0.2,0.3$, and 0.6 are marked with the red dots. (c) the JV curves of $\mathrm{n}-\mathrm{AlGaN} / \mathrm{i}-\mathrm{InGaN} / \mathrm{p}-\mathrm{AlGaN}$ TJs with different In\% and InGaN thicknesses. The current density of the In $\%=0.1$ is small, making the black curve near the $\mathrm{x}$ axis. Inset shows the extracted resistance from the JV curves.

From Fig. 4 (a), the highest $R_{\text {sp }}$ appears in $\operatorname{In} \%=0.3, T_{\text {cr }}=3$ $\mathrm{nm}$, which could be attributed to that the higher tunneling probability is beneficial to the carrier injection into the active region. In $\%=0.1$ has the highest resistance in the TJ, thus leading to the lowest $\mathrm{R}_{\mathrm{sp}}$. For the BJT LEDs, a huge increase of output power and $\mathrm{R}_{\mathrm{sp}}$ from $\mathrm{In} \%=0.1$ to 0.3 could be observed 
in Fig. 4 (b), which results from the enhanced tunneling probability. The output power densities are 1.5, 3365, 19677, and $3033 \mathrm{~W} / \mathrm{m}$ for the In\% equals $0.1,0.2,0.3$, and 0.6, respectively. The extracted data summary is shown in Table SIII in the SM. By proper design of the $\mathrm{n}-\mathrm{AlGaN} / \mathrm{i}-\mathrm{InGaN} / \mathrm{p}$ AlGaN BTJ, the LED performance could be significantly enhanced, which is promising for high-efficiency UV LED. Moreover, the BTJ could also be adopted into the UVB or UVC region by shifting the $\mathrm{Al} \%$ in $\mathrm{AlGaN}$ and optimizing the $\mathrm{In} \%$ and $\mathrm{InGaN}$ thickness.
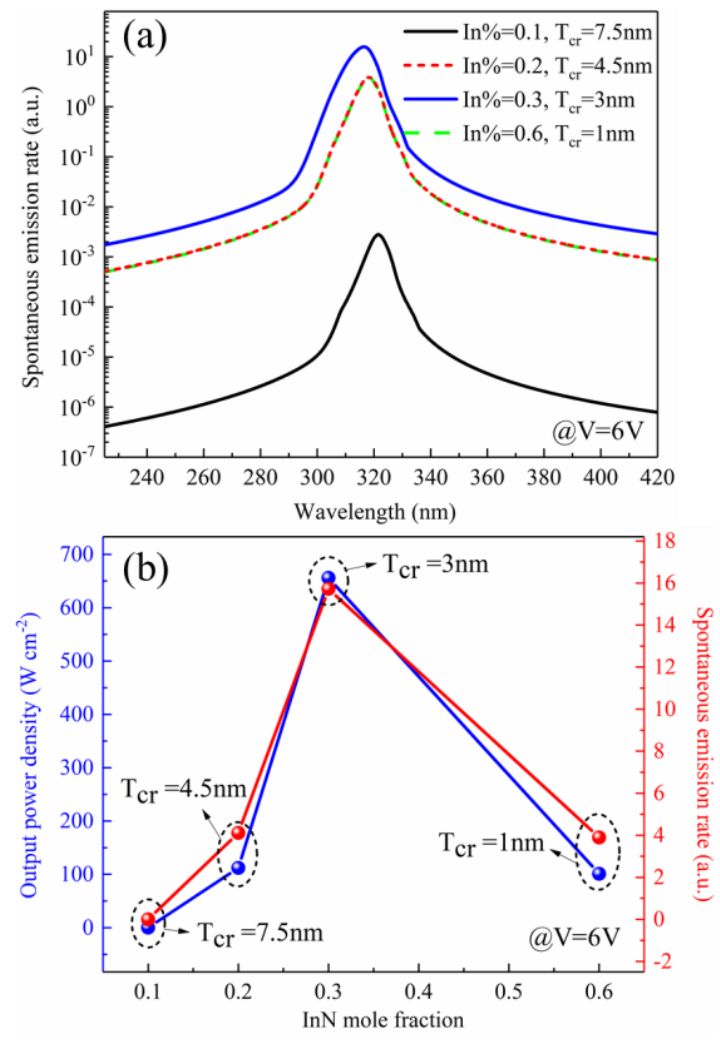

Fig. 4. (a) The $\mathrm{R}_{\mathrm{sp}}$ spectrum with different In $\%$ and InGaN thicknesses. (b) output power density and peak $\mathrm{R}_{\mathrm{sp}}$ of BTJ LEDs for $\mathrm{In} \%=0.1,0.2,0.3$, and 0.6 at $6 \mathrm{~V}$.

In terms of the experimental demonstration, the InGaN would be grown with low temperature, i.e. $600 \sim 750^{\circ} \mathrm{C}$ by MOCVD [20]. It is noted that the Mg memory effect from the bottom p-layer may deteriorate the QW quality, which could be solved by in-situ annealing [11] or growing by MBE [12].

\section{Conclusion}

This study set out to develop the p-side down UV LED by integrating a buried $\mathrm{n}-\mathrm{AlGaN} / \mathrm{InGaN} / \mathrm{p}-\mathrm{AlGaN}$ tunneling junction below the active region to address the insufficient carrier injection issues. By turning over the n-type and p-type regions from the conventional PIN structure, the electron overflow and hole injection could be manipulated due to the desirable polarization-induced potential barriers. Moreover, to investigate the impact of tunneling junction on the carrier transport, In\% from 0.1 to 0.6 and InGaN thicknesses from 2 to $10 \mathrm{~nm}$ are compared in the BTJ LED. In\% and thickness could affect the InGaN potential, and further change the tunneling probability and carrier injection. InGaN thickness which is shorter or longer than $t_{\text {cr }}$ would lower the tunneling probability and deteriorate the LED power. Moreover, by tuning the In\% from 0.1 to 0.6 , the tunneling probabilities increase first and then drop. The optimized $3 \mathrm{~nm} \operatorname{In}_{0.3} \mathrm{Ga}_{0.7} \mathrm{~N}$ tunneling layer contributes to the enhancement of LED output power by several orders of magnitude compared with In\% equals to $0.1,0.2$, and 0.6. The research results represent a further step towards developing highly efficient UV LED.

\section{REFERENCE}

C. Chèze et al., "Luminescent N-polar (In, Ga) N/GaN quantum wells achieved by plasma-assisted molecular beam epitaxy at temperatures exceeding $700^{\circ}$ C," Appl. Phys. Lett., vol. 112, no. 2, p. 022102, 2018.

H. Masui et al., "Luminescence characteristics of N-Polar GaN and InGaN films grown by metal organic chemical vapor deposition," Jpn. J. Appl. Phys., vol. 48, no. 7R, p. 071003, 2009.

H. Turski et al., "Nitride LEDs and Lasers with Buried Tunnel Junctions," Ecs J Solid State Sc., vol. 9, no. 1, p. 015018, 2020. C.-H. Ko et al., "P-down InGaN/GaN multiple quantum wells lightemitting diode structure grown by metal-organic vapor-phase epitaxy," Jpn. J. Appl. Phys., vol. 41, no. 4S, p. 2489, 2002.

$\mathrm{S}$. Newman et al., "Wavelength stable, p-side-down green light emitting diodes grown by molecular beam epitaxy," J. Vac. Sci. Technol., B, vol. 31, no. 1, p. 010601, 2013.

K. Zhang et al., "The properties of reversed polarization yellow InGaN-GaN MQWs in p-side down structure grown by metalorganic chemical vapor deposition on sapphire substrate," Physica E, vol. 64, pp. 57-62, 2014.

C. Forman et al., "Semipolar (2021) III-Nitride P-Down LEDs with in situ anneal to reduce the Mg memory effect," J. Cryst. Growth, vol. 464, pp. 197-200, 2017.

[12] H. Turski, S. Bharadwaj, H. Xing, and D. Jena, "Polarization control in nitride quantum well light emitters enabled by bottom tunneljunctions," J. Appl. Phys., vol. 125, no. 20, p. 203104, 2019.

[13] S. M. Sze and K. K. Ng, Physics of semiconductor devices. John wiley \& sons, 2006.

[14] Z. Ren et al., "III-nitride deep UV LED without electron blocking layer," IEEE Photonics J., vol. 11, no. 2, pp. 1-11, 2019.

[15] S. Krishnamoorthy, F. Akyol, P. S. Park, and S. Rajan, "Low resistance GaN/InGaN/GaN tunnel junctions," Appl. Phys. Lett., vol. 102, no. 11, p. 113503, 2013.

[16] E. Kane, "Zener tunneling in semiconductors," J. Phys. Chem. Solids, vol. 12, no. 2, pp. 181-188, 1960.

[17] S. Krishnamoorthy, D. N. Nath, F. Akyol, P. S. Park, M. Esposto, and S. Rajan, "Polarization-engineered $\mathrm{GaN} / \mathrm{InGaN} / \mathrm{GaN}$ tunnel diodes," Appl. Phys. Lett., vol. 97, no. 20, p. 203502, 2010.

[18] M. Cheong, C. Liu, H. Choi, B. Lee, E.-K. Suh, and H. Lee, "Study of the origin of luminescence in high indium composition InGaN/GaN quantum wells," J. Appl. Phys., vol. 93, no. 8, pp. 46914695, 2003.

[19] P. Mishra et al., "Achieving uniform carrier distribution in MBEgrown compositionally graded InGaN multiple-quantum-well LEDs," IEEE Photonics J., vol. 7, no. 3, pp. 1-9, 2015.

[20] J. Liu et al., "Anomalous indium incorporation and optical properties of high indium content InGaN grown by MOCVD," Journal of Alloys Compounds, vol. 735, pp. 1239-1244, 2018. 